\title{
Role of Pelvic and Para-Aortic Lymphadenectomy in Advanced Ovarian Epithelial Cancer
}

\author{
Ahmed Mohamed Aydarous, Hamed Ahmed Al Azoony, Eid Rizk El Gammal, \\ Ibrahim Hassan Mohamed, Mohamed Foad Abd Elmoty and Mohamed Rashed Abdel Hamid \\ Surgical Oncology Unit, Faculty of Medicine, Al-Azhar University, Cairo, Egypt \\ *Corresponding author: Ahmed Mohamed Aydarous, E-mail: ahmedaydarous343 @ gmail.com
}

\begin{abstract}
Background: the role of detection and removal of retroperitoneal lymph nodes in patients with advanced ovarian cancer disease is unclear. Aim of the work: this study was aimed to evaluate the therapeutic role of pelvic and paraaortic lymphadenectomy in patients with advanced epithelial ovarian cancer.

Patients and Methods: this study was designed as a prospective randomized controlled trial. Thirty patients were divided into two equal groups; 15 patients underwent optimum cytoreduction and in the other 15 patient retroperitoneal lymphadenectomy was added.

Results: the mean number of nodes removed was 26 , and the mean number of positive nodes was 6 . There was significant difference between the two groups of the study when considering operation time (Mean 122 min. Vs. 192 min.). Also there is significant difference between the two groups of the study when considering hospital stay in days (Mean 8 days Vs. 13 days). There was significant difference between the two group of the study when considering the blood loos and need for blood transfusion (26\% Vs. 80\%). There was no significant difference between the two groups of the study when considering recurrence $(73.3 \%$ Vs. 60\%). Conclusion: Our results show improvement towards decrease the recurrence in the lymphadenectomy group but with non-significant results.
\end{abstract}

Keywords: Advanced epithelial ovarian cancer, Prognostic factors, Survival, Therapeutic role pelvic and aortic lymphadenectomy.

\section{INTRODUCTION}

Worldwide, ovarian cancer is the seventh most common cancer in women and the second most common gynecologic cancer, after endometrial cancer. Ovarian cancer is usually seen in perimenopausal women with mean age being 63 years ${ }^{(1)}$. Ovarian epithelial carcinoma (OECs), the most common ovarian malignancy, is a heterogeneous disease with several histologic subtypes that show characteristic cytogenetic features, molecular signatures, oncologic signaling pathways and clinical-biologic behavior ${ }^{(2)}$. OECs are notoriously difficult to diagnose in the early stages. Even patients with advanced disease may present with nonspecific abdominal and pelvic symptoms. Also, there are no reliable biomarkers that may aid in early detection $^{(3)}$.

The contribution of imaging includes surgery planning and selection of candidates for neoadjuvant chemotherapy by demonstration of non-optimally resectable ${ }^{(4)}$.

Surgery is the primary treatment of choice for all ovarian cancers. Radical debulking includes bilateral salpingo-oophorectomy, hysterectomy, infracolic omentectomy, and peritoneal surface biopsy and washing. In patients with extraovarian disease, extent of surgery is individualized. Tumor resectability is of great concern in stage III and IV disease ${ }^{(5)}$.Due to nonspecific symptoms, lack of reliable biomarkers, frequent diagnosis of advanced disease and the presence of drugresistant histologic types, the long-term cure rates for ovarian epithelial carcinoma is limited, for these reasons OECs are the most lethal gynecologic malignancy. Approximately $70 \%$ of women with OECs have advanced disease at presentation, and $65 \%$ of women die within 5 years of diagnosis ${ }^{(6)}$. The value of pelvic and paraaortic lymphadenectomy in advanced ovarian tumors is still in debate. It needs more studies to be established, understood, and best of all tailored to the population that would have the most benefits in relation to the cost ${ }^{(7)}$.

\section{AIM OF THE WORK}

This study was aimed to evaluate the therapeutic role of pelvic and para-aortic lymphadenectomy in patients with advanced epithelial ovarian cancer.

\section{PATIENTS AND METHODS}

This prospective study included thirty female patients presented by pelvi-abdominal swelling and elevated CA-125 suggesting ovarian cancer. All patients underwent primary surgery in the Surgical Oncology ward of Al-Azhar university hospitals from May 2016 to January 2018. The study was approved by the Ethics Board of Al-Azhar University and an informed written consent was taken from each participant in the study.

\section{- Inclusion criteria:}

Patients were only included in this study if they had all of the following criterion: Advanced ovarian epithelial cancer stage (III and IV).

- Exclusion criteria: Patients were excluded if they had any of the following criteria: Synchronous abdominal or pelvic pathology, medically unfit for surgery and residual tumor more than $1 \mathrm{~cm}$ after surgery.

- Patient consent: Patients agreed to be included in the study and an informed consent was taken. 


\section{Surgical technique}

All patients were undergone standard longitudinal laparotomy then further divided to two groups randomly:

- Group (A): Maximal surgical effort for Optimum surgical procedures included all of the following: Total abdominal hysterectomy, Bilateral salpingooophorectomy, Infracolic omentectomy, Appendectomy, Surgical removal of all tumor masses and additional surgery (e.g. intestinal resections) if required.

- Group (B): Optimum surgical procedures as group (A) in addition to lymphadenectomy in form of systematic pelvic lymphadenectomy to the level of aortic bifurcation but if there is palpable paraortic lymph nodes, systemic paraortic lymphadenectomy

\section{RESULTS}

We included 15 cases in each group. The demographic data of these patients were summarized in Table (1).

Table (1): Comparison between groups according to demographic data.

\begin{tabular}{|c|c|c|c|c|c|}
\hline & \multicolumn{2}{|c|}{ No LNDs Dissection Arm (A) } & \multicolumn{2}{|c|}{ LNDs Dissection arm (B) } \\
\hline & & $\mathrm{N}$ & $\%$ & $\mathrm{~N}$ & $\%$ \\
\hline \multirow{3}{*}{ Age Groups } & $50 \sim 60$ Years & 8 & $53.3 \%$ & 4 & $26.7 \%$ \\
\hline & $60 \sim 70$ Years & 4 & $26.7 \%$ & 6 & $40.0 \%$ \\
\hline & $70 \sim 80$ Years & 3 & $20.0 \%$ & 5 & $33.3 \%$ \\
\hline \multirow{2}{*}{ CA 125 Groups } & $<500$ & 9 & $60.0 \%$ & 8 & $53.3 \%$ \\
\hline & $>500$ & 6 & $40.0 \%$ & 7 & $46.7 \%$ \\
\hline \multirow{2}{*}{ Ascitis Groups } & $<1000$ & 7 & $46.7 \%$ & 10 & $66.7 \%$ \\
\hline & $>1000$ & 8 & $53.3 \%$ & 5 & $33.3 \%$ \\
\hline \multirow{5}{*}{ Histopahhological type } & serous & 12 & $80.0 \%$ & 10 & $66.7 \%$ \\
\hline & endometroid & 1 & $6.7 \%$ & 2 & $13.3 \%$ \\
\hline & mucinous & 1 & $6.7 \%$ & 2 & $13.3 \%$ \\
\hline & other & 1 & $6.7 \%$ & 1 & $6.7 \%$ \\
\hline & clear cell & 0 & $0.0 \%$ & 0 & $0.0 \%$ \\
\hline \multirow{2}{*}{ Stage } & Stage 3 & 11 & $73.3 \%$ & 10 & $66.7 \%$ \\
\hline & Stage 4 & 4 & $26.7 \%$ & 5 & $33.3 \%$ \\
\hline \multirow{3}{*}{ Grade } & Grade 1 & 0 & $0.0 \%$ & 0 & $0.0 \%$ \\
\hline & Grade 2 & 9 & $60.0 \%$ & 6 & $40.0 \%$ \\
\hline & Grade 3 & 6 & $40.0 \%$ & 9 & $60.0 \%$ \\
\hline
\end{tabular}

Table (2): Lymph nodes status of treatment arm

\begin{tabular}{|l|l|l|l|l|}
\hline \multirow{2}{*}{} & \multicolumn{2}{|l|}{ LNDs Dissection Arm (B) } & P-value \\
\cline { 2 - 5 } & Mean & Maximum & Minimum & \\
\hline Number of LNs removed & 26 & 40 & 18 & 0.00 \\
\hline Number of LNDs positive for malignancy & 6 & 13 & 0 & 0.00 \\
\hline
\end{tabular}

Tables (2) Describes the number, mean , maximum and minimum of lymph nodes resected by treatment arm, also number of lymph nodes which were found positive for metastasis.

Table (3): Compares the two groups of the study when considering operation time and hospital stay in days

\begin{tabular}{|l|l|l|l|l|l|l|c|}
\hline & \multicolumn{2}{|l|}{ No LND Dissection Arm (A) } & \multicolumn{2}{l|}{ LNDs Dissection Arm (B) } & P-value \\
\cline { 2 - 8 } & Mean & Maximum & Minimum & Mean & Maximum & Minimum & \\
\hline Operation time & 122.33 & 180.0 & 90.0 & 192.67 & 280.0 & 120.0 & 0.00 \\
\hline Hospital Stay in Days & 8 & 10 & 5 & 13 & 17 & 9 & 0.00 \\
\hline
\end{tabular}

There is significant difference between the two group of the study when considering operation time and Hospital Stay in Days (P is 0.00) Table (3). 
ejhm.journals.ekb.eg

Table (4): Compares the two groups of the study when considering the blood loss and need for blood transfusion.

\begin{tabular}{|l|l|l|l|l|l|l|}
\hline \multicolumn{2}{|c|}{} & \multicolumn{2}{|l|}{$\begin{array}{l}\text { No LNDs } \\
\text { Dissection Arm (A) }\end{array}$} & \multicolumn{2}{l|}{$\begin{array}{l}\text { LNDs Dissection Arm } \\
\text { (B) }\end{array}$} & P-value \\
\cline { 3 - 7 } & N & $\%$ & N & $\%$ & \\
\hline \multirow{3}{*}{ Blood Transfusion } & $\begin{array}{l}\text { No need For } \\
\text { Transfusion }\end{array}$ & 11 & $73.3 \%$ & 3 & $20.0 \%$ & \multirow{2}{*}{0.002} \\
\cline { 2 - 5 } & $\begin{array}{l}\text { Needed Blood } \\
\text { Transfusion }\end{array}$ & 4 & $26.7 \%$ & 12 & $80.0 \%$ & \\
\hline
\end{tabular}

There is significant difference between the two groups of the study when considering the blood loos and need for blood transfusion Table (4).

Table (5): Compares the two groups of the study when considering the recurrence.

\begin{tabular}{|c|c|c|c|c|c|c|}
\hline & \multicolumn{2}{|c|}{$\begin{array}{l}\text { No LNDs Dissection } \\
\text { Arm (A) }\end{array}$} & \multicolumn{2}{|c|}{$\begin{array}{l}\text { LNDs Dissection Arm } \\
\text { (B) }\end{array}$} & P-value \\
\hline & & $\mathrm{N}$ & $\%$ & $\mathrm{~N}$ & $\%$ & \multirow{3}{*}{0.456} \\
\hline \multirow{2}{*}{ Recurrence } & No recurrence & 4 & $26.7 \%$ & 6 & $40.0 \%$ & \\
\hline & Recurrence & 11 & $73.3 \%$ & 9 & $60.0 \%$ & \\
\hline
\end{tabular}

There is no significant difference between the two group of the study when considering recurrence $(\mathrm{P}>0.05)$ Table (5).

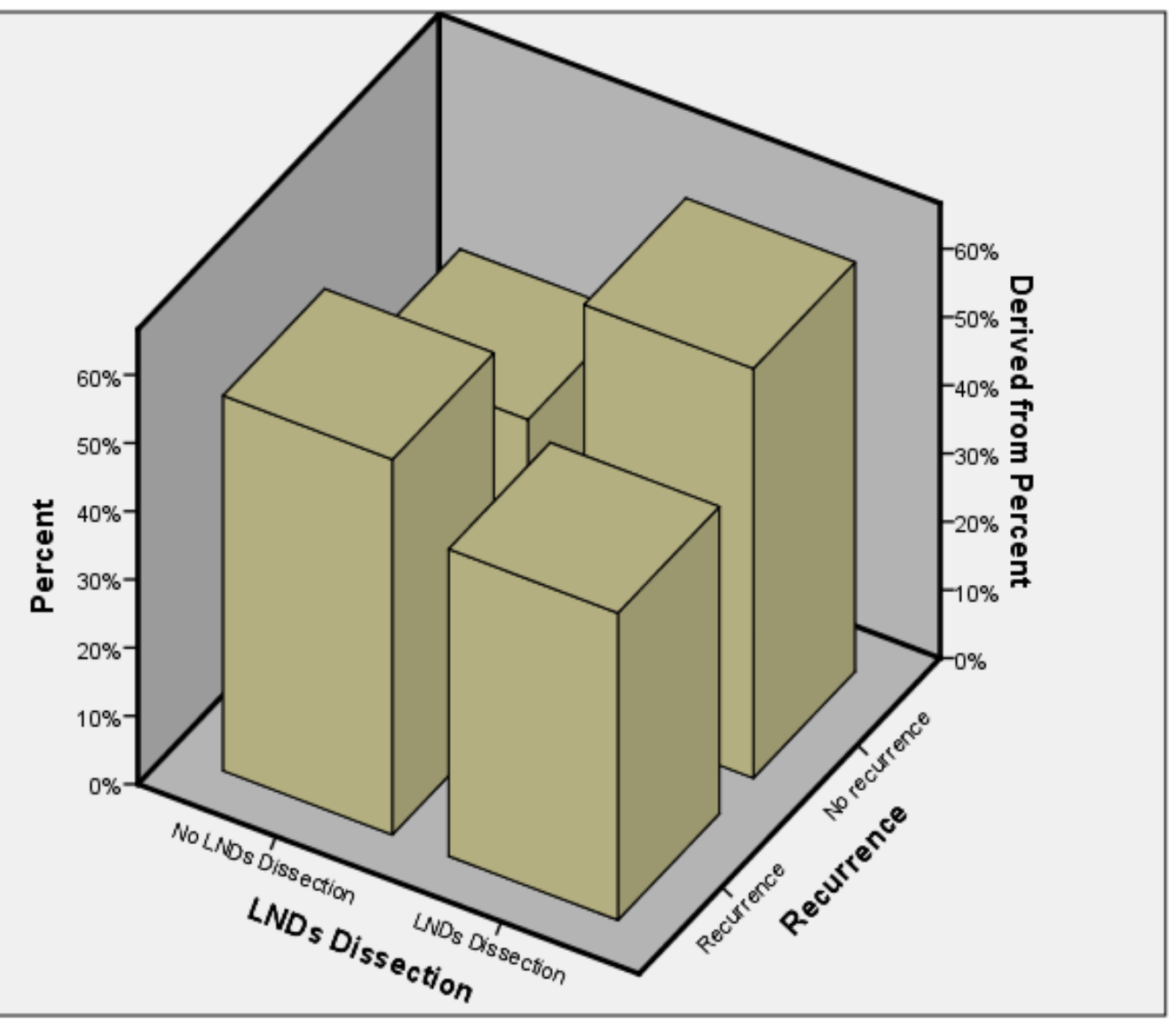

Figure (1): Bar graph compares the two group of the study when considering the recurrence. 


\section{DISCUSSION}

The role of systematic lymphadenectomy in advanced stages of ovarian cancer is somewhat unclear. Because lymphatic spread is a common feature of advanced epithelial ovarian cancer and nodal positivity cannot be diagnosed reliably either with imaging or by intraoperative palpation. The observed rate of positive lymph nodes depends essentially on the completeness of lymphadenectomy (8).

Our hypothesis depends on that in patients with macroscopic complete intraperitoneal debulking, systematic lymphadenectomy might theoretically add complete resection of retroperitoneal disease, thus achieving a true macroscopic complete resection, and especially we know that lymph nodes have poor blood supply.

Surgical management of patients with ovarian cancer is challenging, and systematic lymphadenectomy is a major surgical procedure. This study provides evidence that the procedure itself is feasible in the framework of randomized clinical trial. Women who underwent systematic lymphadenectomy had a higher incidence of postoperative complications, but almost all of these were mild, consisting of lymphocysts or lymphedema. The mean operating time was about 192 minutes longer and the blood loss higher in the lymphadenectomy arm than in the control arm, more patients underwent a blood transfusion in the experimental group. Also, there was prolonged duration of postoperative hospital stay in the lymphadenectomy arm, and no perioperative deaths occurred.

Our study demonstrated that there is no significant difference between the two groups of the study when considering recurrence.

Kim et al. ${ }^{(9)}$ have performed a meta-analysis for comparing the efficacy for OS between Systemic lymphadenectomy and unsystematic lymphadenectomy. They analyzed 9 studies (2 randomized controlled trials and 7 observational studies) with 21,919 patients with EOCs who underwent staging laparotomy, including Systemic lymphadenectomy and unsystematic lymphadenectomy. Although this meta-analysis suggests the possibility that Systemic lymphadenectomy may be has a role in improving OS in patients with advanced-stage EOCs. Because Systemic lymphadenectomy may contribute to the detection of occult lymph node metastasis with chemoresistance.

In a study of Pereira et al. ${ }^{(10)}$ on 116 consecutive cases with primary EOCs and stage IIIC or IV disease having nodal metastasis and treated at Mayo Clinic in Arizona, Florida, and Rochester, Minnesota between 1996 and 2000.He addressed a survival benefit of lymphadenectomy in the patients with EOCs with advanced peritoneal disease of more than $2 \mathrm{~cm}$, when more than 40 lymph nodes were resected. There was a survival benefit in a small subset of patients with advanced peritoneal disease of more than $2 \mathrm{~cm}$ and no residual disease after debulking when more than 10 positive nodes were resected, although the number of patients is too low to establish a valid conclusion.

In a study of Sakai et al. ${ }^{(11)}$ on hundred and eighty patients with histologically proven and optimally debulked (residual tumor $<1 \mathrm{~cm}$ ).EOCs diagnosed between 1986 and 2009 were registered and treated by the Tokai Ovarian Tumor Study Group, consisting of Nagoya University Hospital and affiliated hospitals. All patients were divided into two groups as follows: Group A patients underwent optimal cytoreductive surgery and Groups B Patients underwent systematic retroperitoneal lymphadenectomy in addition to cytoreductive surgery. In conclusion, data suggested that there were no significant differences in the OS and PFS between the systematic retroperitoneal lymphadenectomy and control groups.

Chang et al. ${ }^{(12)}$ has studded data of 189 consecutive patients with FIGO stage IIIC ovarian cancer between 2000 and 2011, who underwent primary cytoreductive surgery followed by platinumand taxane-based chemotherapy. All patients were classified into two groups: patients who underwent systematic pelvic and para-aortic lymphadenectomy and who underwent cytoreduction only. The results, showed that patients who underwent systematic lymphadenectomy had significantly improved PFS and OS. In patients with no gross residual disease or residual disease $0.1-1 \mathrm{~cm}$, the median OS time of those who had lymphadenectomy was significantly longer than those who did not.But, in patients with residual disease $>1 \mathrm{~cm}$, there was no significant difference in OS according to lymphadenectomy. The study concluded, that systematic lymphadenectomy may have a therapeutic value and be significantly associated with improved survival in stage IIIC ovarian cancer patients with grossly no visible residual disease.

Gao et al. ${ }^{(13)}$ has conducted a literature search of the Pubmed, Embase and Cochrane Library databases was performed up to 2014. Fourteen relevant studies including 3488 subjects were included in the analysis. They concluded that lymphadenectomy can improve the five year overall survival rate in advanced stage epithelial ovarian cancer but not in early stage epithelial ovarian cancer, or in patients with residual tumor more than $2 \mathrm{~cm}$.

In summary, we did not think that our limited results would immediately lead to the possibility of omitting lymphadenectomy. As the indication for systemic retroperitoneal lymphadenectomy is still 
controversial, considering the adverse effects and possible survival benefits, we hope that further investigation involving a larger-scale, prospective, clinical study will be conducted in the future.

\section{CONCLUSION}

The results of the present study identified statistically significant differences between lymphadenectomy and non- lymphadenectomy groups when considering operation time, need for blood transfusion and hospital stay in days. Also there is improvement towards decrease the recurrence in the lymphadenectomy group but with non-significant results. However, generalization of this recommendation needs a multi-institutional widebased study.

\section{RECOMMENDATION}

It is recommended that further studies should be conducted to assess the role of HIPEC for completion of cytoreduction and elimination of peritoneal residual.

\section{REFERENCES}

1. Torres-Mejía G, Royer R, Lacuachaqui M, Akbari MR, Giuliano AR, Martínez-Matsushita L, Phelan CM (2015): Recurrent BRCA1 and BRCA2 mutations in Mexican women with breast cancer. Cancer Epidemiology and Prevention Biomarkers, 24(3): 498-505.

2. Chui MH, Gilks CB, Cooper K, Clarke BA (2013): Identifying Lynch syndrome in patients with ovarian carcinoma: the significance of tumor subtype. Advances in anatomic pathology, 20(6): 378-386.

3. Ledermann JA, Raja FA, Fotopoulou C, GonzalezMartin A, Colombo N, Sessa C, ESMO Guidelines Working Group. (2013): Newly diagnosed and relapsed epithelial ovarian carcinoma: ESMO Clinical Practice Guidelines for diagnosis, treatment and follow-up. Annals of oncology, 24(6):24-32.
4. Kyriazi S, Collins DJ, Morgan VA, Giles SL, deSouza NM (2010): Diffusion-weighted imaging of peritoneal disease for noninvasive staging of advanced ovarian cancer. Radiographics, 30(5): 1269-1285.

5. Elattar A, Bryant A, Winter-Roach BA, Hatem M, Naik R (2011): Optimal primary surgical treatment for advanced epithelial ovarian cancer. https://www.ncbi.nlm.nih.gov/pubmed/21833960

6. Devan SM, Pailoor J, Sthaneshwar P, Narayanan V (2013): Pattern of tissue expression of CA-125 and HE4 in primary epithelial ovarian tumours and correlation with serum CA-125 levels. Asian Pacific Journal of Cancer Prevention, 14(8): 4545-4548.

7. Al Rawahi T, Lopes AD, Bristow RE, Bryant A, Elattar A, Chattopadhyay S and Galaal K (2013): Surgical cytoreduction for recurrent epithelial ovarian cancer. Cochrane Database Syst Rev., 2:CD008765.

8. Harter P, Gnauert K, Hils R et al. (2007): Pattern and clinical predictors of lymph node metastases in epithelial ovarian cancer. Int J Gynecol Cancer , 17:1238-1244.

9. Kim HS, Ju W, Jee BC, Kim YB, Park NH et al. (2010): Systematic lymphadenectomy for survival in epithelial ovarian cancer: a meta-analysis. International Journal of Gynecological Cancer, 20(4): 520-528.

11. Pereira A, Pérez-Medina T Magrina JF, Magtibay PM, Millan I et al. (2012): The role of lymphadenectomy in node-positive epithelial ovarian cancer. International Journal of Gynecological Cancer, 22(6): 987-992.

12. Sakai K, Kajiyama H, Umezu T, Shibata K, Mizuno M et al. (2012): Is there any association between retroperitoneal lymphadenectomy and survival benefit in advanced stage epithelial ovarian carcinoma patients? Journal of Obstetrics and Gynaecology Research, 38(7):1018-1023.

13. Chang SJ, Bristow RE, Ryu HS (2012): Prognostic significance of systematic lymphadenectomy as part of primary debulking surgery in patients with advanced ovarian cancer. Gynecologic oncology, 126(3): 381-386.

14. Gao J, Yang $X$, Zhang $Y$ (2014): Systematic lymphadenectomy in the treatment of epithelial ovarian cancer: a meta-analysis of multiple epidemiology studies. Japanese journal of Clinical Oncology, 45(1):49-60. 